\title{
A Novel Pyroptosis-related Genes Prognostic Signature Affects the Immune Infiltration Patterns in Hepatocellular Carcinoma
}

Yiqun Jin ( $\sim$ dr_jyq@163.com )

Affiliated Hangzhou First People's Hospital Zhejiang University School of Medicine: Hangzhou First People's Hospital https://orcid.org/0000-0001-6505-6206

Bai. Xue-song

Chinese Academy of Medical Sciences \& Peking Union Medical College

\section{Research Article}

Keywords: Pyroptosis-related genes, Prognosis, Hepatocellular carcinoma, Inflammatory response, Immunotherapy

Posted Date: November 1st, 2021

DOI: https://doi.org/10.21203/rs.3.rs-1020197/v1

License: (c) (i) This work is licensed under a Creative Commons Attribution 4.0 International License. Read Full License 


\section{Abstract}

\section{Purpose}

Pyroptosis is an inflammatory form of cell death associated with tumorigenesis and progression. However, the prognostic value of pyroptosis-related genes (PRGs) in hepatocellular carcinoma (HCC) have not been elucidated.

\section{Methods}

We downloaded mRNA expression profiles and clinical information from TCGA and ICGC database. Then, differently expressed PRGs were screened to construct a multigene prognostic signature by least absolute contraction and selection operator (LASSO) Cox regression method in TCGA cohort. Date from ICGC was used to validate the robustness of this signature. Kaplan-Meier analysis was used to compare overall survival (OS) between high- and low-risk group. Univariate and multivariate Cox analysis were performed to identify the independent prognostic value of the signature. Gene set enrichment analysis (GSEA) was utilized to conduct GO and KEGG analysis. Single-sample gene set enrichment analysis was implemented to assess the immune cell infiltration and immune-related function. TIDE algorithm evaluated the significance of this signature in predicting immunotherapeutic sensitivity.

Results

An 8-PRGs prognostic model was established. The OS of low-risk group was significantly increased compared to high-risk group. Receiver operating characteristic curve showed the model had a good prognostic predictive accuracy. Cox regression analysis proved the model an independent predictor for OS in HCC. GSEA indicated that the risk score was associated with immune response. Furthermore, different subgroups exhibited different immunoinfiltration patterns, different immune-checkpoint levels and different potential responses for immune-checkpoint blockade therapy.

\section{Conclusion}

An 8-PRGs signature can predict the prognosis of HCC patients and may act as an immunotherapeutic potential target for HCC.

\section{Introduction}

Hepatocellular carcinoma (HCC) is one of the most common serious life-threatening malignancies with a high cancer-related mortality rate throughout the world ${ }^{[1][2]}$. Despite the continuous development of treatments for HCC, the improvement of survival rate is still limited due to the difficulty in early diagnosis and high recurrence rate ${ }^{[3]}$. Therefore, it is important to explore better methods for early diagnosis and prognostic evaluation to geared towards ameliorating the treatment and prognosis of HCC patients. 
Pyroptosis is a new form of programmed cell death, also known as cellular inflammatory necrosis, characterized with release of inflammatory cytokines and characteristic bubble-like protrusions ${ }^{[4]}$ [5]. Gasdermin family proteins, including gasdermin-A to gasdermin-E and PJVK are the main executors of pyroptosis. Caspases play a central role in the assembly of inflammasomes and the initiation of pyroptosis. Through the canonical and non-canonical pathways, specific caspase family proteins are activated, and cleave GSDMD, GSDMB or GSDME at the intramolecular binding between the N-terminal effector domain and $\mathrm{C}$-terminal inhibitory domain ${ }^{[6][7]}$. The cytotoxic $\mathrm{N}$-terminal of gasdermin then oligomerizes and forms a 10 to $20 \mathrm{~nm}$ transmembrane pore, which promotes the maturation and release of pro-inflammatory cytokines (such as IL-1 $\beta$ and IL-18). Then it causes the infiltration of immune cells and inflammation in the microenvironment, which in turn leads to cell swelling and plasma-membrane rupture $^{[8]}$. In addition to caspase, granzyme proteases from lymphocytes and natural killer cells can also mediate the cleavage of gasdermin and induce the pyroptosis of cells ${ }^{[9]}$.

Pyroptosis was first studied in the immune defense against pathogen infection ${ }^{[10]}$. Recently, increasing evidence indicated pyroptosis a dual role of promoting and inhibiting in the pathogenesis of tumors ${ }^{[11]}$. On the one hand, it can effectively kill tumor cells by inducing apoptosis. On the other hand, with arousing inflammatory response, pyroptosis cause a suitable microenvironment for the malignant transformation and growth of tumor ${ }^{[5]}$. However, the role of pyroptosis in HCC and its rele in prognosis of HCC deserve further study.

In this study, we constructed a prognostic model based on pyroptosis related genes (PRGs). Then, we performed functional enrichment analysis to explore its underlying mechanism and the correlation with the tumor immune microenvironment. Collectively, our study aims to provide a new understanding of the role of pyroptosis in HCC, and explore the prognostic value of PRGs for HCC patients.

\section{Materials And Methods}

\section{Data and information collection}

We downloaded the total mRNA sequencing data and corresponding clinicopathologic information of $374 \mathrm{HCC}$ and 50 normal tissues from the The Cancer Genome Atlas (TCGA) database on July 28, 2021 (https://portal.gdc.cancer.gov/). Another 231 patients' mRNA sequencing data and corresponding clinical information was obtained from the International Cancer Genome Consortium (ICGC) database for external validation cohort (https://dcc.icgc.org/projects/LIRI-JP). The involved data from these databases are open-access and our study abide by the data acquisition policies and publication guidelines of them.

\section{Identifying the differentially expressed PRGs}

We collected 90 genes related to pyroptosis from previously published reviews ${ }^{[4][12]-[24]}$, as shown in Supplementary Table 1. The mRNA sequencing data for the differentially expressed PRGs between 
tumor and normal tissues were identified using "limma" R package in R software 4.1.0 with a $p$ value $<0.05$ for subsequent analysis. The "pheatmap" and "ggplot2" R packages were employed to drawn heatmaps, volcano plots and box plots.

\section{Construction and validation of prognostic risk model based on PRGs}

Cox regression analysis was used to assess the association between each PRG and survival status to assess the prognostic value of PRGs. Further, the least absolute shrinkage and selection operator (LASSO) -penalized Cox regression analysis were conducted using "GLMnet" R package to narrow the range of candidate genes and construct a prognostic model. The penalty parameter $(\lambda)$ was determined and followed the minimum criterion (in other words, the $\lambda$ value corresponds to the lowest partial likelihood deviation). Calculate the risk score using the following formula: risk score $=\sum_{i=1}^{n} X_{i} \times Y_{i}$, where $X$ represented the expression level of each retained PRGs and $Y$ represented the corresponding regression coefficient. The mutation profiles of each gene involved in the risk model were analyzed in HCC via cBioPortal database (http://cbioportal.org) ${ }^{[25]}$. The median value of risk score was used to divide the HCC patients into high-risk and low-risk groups. "Rtsne" and "ggplot2" R packages were utilized to perform principal component analysis (PCA) and t-distributed stochastic neighbor embedding (t-SNE) analysis for dimensionality reduction and verifying the distribution of different groups. Kaplan-Meier survival analysis was conducted to compare the overall survival (OS) between two subgroups. R packages "survival", "survminer", "survivalROC" and "timeROC" were employed to generate time-dependent ROC curve analysis. Univariate and multivariate Cox regression analysises were used for determination the independent prognostic value of the 8-gene model.

\section{Gene set enrichment Analysis and Immune Infiltration Analysis}

To explore biological functions and pathways affected by the risk scores, gene sets database "c2.cp.kegg.v7.4.symbols.gmt" and "c5.go.v7.4.symbols.gmt" were used to conduct gene set enrichment analysis via the software Gene Set Enrichment Analysis (GSEA, version 4.0.1) ${ }^{[26][27]}$. The FDR value q and normalized value $p<0.05$ were considered statistically significant.

\section{Immune Function Analysis and Immunotherapy prodiction}

Single-sample gene set enrichment analysis (ssGSEA) was performed with "GSVA", "ESTIMATE" and "GSEABase" R packages to score the immune functions, pathways, as well immune cells and stromal cells infiltration level, for each sample. Tumor immune dysfunction and rejection algorithm (TIDE, http://tide.dfci.harvard.edu) was used to compare the therapeutic effect evaluation of immune checkpoint blockade (ICB) in different groups.

\section{Statistical analysis}

The WilCoxon test were used to compare differences in gene expression between tumor and normal tissue and immune scores between high- and low risk groups. The two-sided log-rank test was utilized to 
perform Kaplan-Meier survival analysis for compare the OS of patients between two subgroups. Spearman correlation analysis was used to test the correlation between immune scores and risk scores of the prognosis risk model. All statistical analyses were performed with R software (V4.1.0)

\section{Results}

\section{Identification of differentially expressed PRGs.}

First, the transcriptional profiles of 90 PRGs were investigated in the TCGA mRNA-seq data for 50 normal tissues and $374 \mathrm{HCC}$ tissues. A total of 65 differentially expressed PRGs were screened out (All $p<0.05$ ). Among them, 58 genes were enriched in HCC tissues while the 7 other genes were downregulated compared to normal tissues. The mRNA expression levels of these genes are presented in the form of volcano map and heatmap (Figure 1A, B). The correlation network of these differentially expressed PRGs was shown in the Figure $1 \mathrm{C}$.

\section{Classification of HCC based on differentially expressed PRGs}

A consistent cluster analysis using the data of $374 \mathrm{HCC}$ cases from TCGA database was preformed to explore the association between the transcriptional profiles of 65 PRGs and HCC subtypes. We increased the clustering variable $(\mathrm{k})$ from 2 to 9 and found that the intra-group correlations were highest and the inter-group correlations were low when $k=2$, suggesting that $374 \mathrm{HCC}$ patients could be divided into two clusters according to the expression of 65 PRGs (Figure 2A). Then we preformed PCA analysis to verify the discrete distribution of the two clusters (Supplementary Figure 1). Kaplan-Meier curves showed that OS of cluster 1 was significantly better than that of cluster 2 (Figure 2B $p=0.004872$ ). Heatmap was drawn to show the relationship between the expression profiles of 65 PRGs and clinical features, such as tumor size (T1-4), lymph node metastasis(N0-1), distant organ metastasis(M0-1), clinical stage (stage IIV), histologic grading (G1-G4), age ( $\leq 60$ or $>60$ years), gender (female or male) and survival status (alive or dead) (Figure 2C). It could be observed that there were significant differences in tumor size and clinical stage between the two clusters (Figure 2D, Figure 2E, both $p<0.001$ ).

\section{Construction of prognostic risk model according to PRGs}

HCC samples with incomplete clinical information were removed and survival-related PRGs were screened by univariate COX regression analysis. The results revealed that a total of 11 PRGs (CASP8, CHMP3, HSP90AA1, BAK1, HSP90AB1, GSDMC, GSDME, STK4, CGAS, NOD2) were identified correlated with OS $(p<0.01)$ and retained for further analysis (Figure $3 A)$. LASSO-Cox regression analysis was preformed to construct an 8 genes prognostic model (HSP90AA1, CHMP4B, BAK1, HSP90AB1, GSDMC, GSDME, STK4, NOD2) based on the optimal value of penalty parameter $(\lambda)$ (Figure 3B, $C$ ), the expression of which were upregulated in tumor tissue and correlated with poor prognosis according to Gene Expression Profiling Interactive Analysis (GEPIA) (Supplementary Figure 2). The risk score was calculated as follows: Risk Score $=(0.309 * H S P 90 A A 1 \exp )+.(0.131 *$ CHMP4B exp. $)+(0.051 * B A K 1$ exp. $)+$ $(0.006 *$ HSP90AB1 exp. $)+(0.173 *$ GSDMC exp. $)+(0.236 *$ GSDME exp. $)+(0.066 *$ STK4 exp. $)+$ 
$(0.107 *$ NOD2 exp.). Based on the median value of risk score calculated by the formula, HCC patients in TCGA cohort were divided into low-risk and high-risk subgroups (Figure 3D). The t-SNE analysis and PCA showed that patients with different risks were well distributed into two directions (Figure 3E). In addition, the scatter plot shows that high-risk patients had more deaths and shorter survival time than low-risk patients (Figure 3F). Consistently, the Kaplan-Meier curve showed that high-risk patients had significantly poorer OS than low-risk patients (Figure 3G). Time-dependent ROC curves were generated for evaluation of the sensitivity and specificity of the prognostic model and the area under the curve (AUC) reached 0.717 at 1 year, 0.665 at 2 years, and 0.658 at 3 years (Figure $3 \mathrm{H}$ ).

\section{External Validation of the 8-Gene risk Signature}

As the external validation set for the prognostic model, patients in the ICGC cohort were also divided into high-risk or low-risk groups based on the median risk score calculated by the same equation (Figure 4A). PCA and t-SNE analyses confirmed a satisfactory separation of patients between the two subgroups (Figure 4B, C). As shown in Figure 4D to 4F, the OS and ROC analyses of these two subgroups showed similar results to the TCGA cohort.

\section{Prognostic Value of the Prognostic Risk Model}

Univariate and multivariate Cox regression analyses were preformed to assess independent predictive value of the risk scores derived from 8-genes model for OS of HCC patients. Univariate Cox regression analysis revealed that risk score was negatively correlated with prognosis, with a hazard ratio 3.303 (Figure 5A, $p<0.001$ ). Further, multivariate analysis showed that risk score was one of independent factors for predicting the prognosis in $\mathrm{HCC}$ (Figure $5 \mathrm{~B}, p<0.001$ ). Data from the ICGC validation set also confirm this result (Supplementary Figure 3). In addition, heatmap of PRGs expression and clinical characteristics based on the TCGA data showed significant differences in age, survival status, tumor size, and clinical stage between the low-risk and high-

risk subgroups (Figure 5C). Correspondingly, the risk scores in histologic grading 3-4 and clinical stage IIIIV were significantly elevated compared to histologic grading 1-2 and clinical I-II (Figure 5D, $p=0.00015$, $p=0.013$ ). The area under the ROC curve showed good predictive accuracy of the PRGs model for prognosis in $\mathrm{HCC}$, and could provide more accurate predictive value when combined with tumor stage (Figure 5E, AUC=0.733).

\section{Expression and Mutation profiles of Prognostic Genes Between HCC Tissues and Para-carcinoma Tissues.}

Immunohistochemistry from the Human Protein Atlas (HPA) database for the prognostic PRGs showed that the expression in cancer tissues were mostly higher than that of normal tissues (Figure 6A).

Furthermore, we analyzed the expression and mutation profiles of the 8 PRGs based on the HPA database and cBioProtal database. Data form the 353 samples with mutation and Copy Number Alterations (CNA) showed that 67 patients (19\%) had heterogeneous mutations (Figure $6 \mathrm{~B}$ ). Thereinto, $0.57 \%$ patients had 
deep deletion, $0.85 \%$ patients had missense mutation and $0.28 \%$ patients had truncating mutation in HSP90AA $1 ; 0.85 \%$ patients had amplification while $0.57 \%$ patients had structural variant in CHMP4B; $1.42 \%$ patients had amplification BAK $1 ; 3.12 \%$ patients had amplification, $0.28 \%$ patients had truncating mutation and $0.57 \%$ patients had missense mutation in HSP90AB $1 ; 10.2 \%$ patients had amplification, $1.13 \%$ patients had missense mutation and $0.28 \%$ patients had deep deletion in GSDMC; $0.28 \%$ patients had amplification and truncating mutation respectively, while $0.57 \%$ patients had missense mutation in GSDME; $0.28 \%$ patients had missense mutation STK4; $0.28 \%$ patients had amplification and deep deletion respectively, while $1.42 \%$ patients had missense mutation in NOD2.

\section{Analysises of Biological Function and Pathway.}

In order to further explore the biological processes and pathways through which the 8-gene signature affect the prognosis in HCC, GSEA were performed to conduct Gene Ontology (GO) and Kyoto Encyclopedia of Genes and Genomes (KEGG) analyses between the high- and low-risk groups. 20 GO functions and 20 KEGG pathways were respectively enriched in the high-risk group with the FDR value $q$ and normalized value $p<0.05$. The results turned out that some functions and pathway involved in regulation of cell cycle phase transition were enriched in the high-risk group. And in addition to this, several tumor-associated pathways were significantly enriched in high-risk group, including apoptosis, cell cycle, WNT, TGF- $\beta$, VEGF and MAPK signaling pathway. Interestingly, the high-risk group was significantly correlated with some pathways related with immune response, including FC- $y$ receptor mediated phagocytosis, nod like receptor signaling pathway, T cell receptor signaling pathway, B cell receptor signaling pathway, toll like receptor signaling pathway, natural killer cell mediated cytotoxicity, mTor signaling pathway (Figure 7A and 7B).

\section{Comparison of Immune status between subgroups}

Since the relevance between the risk score and immune response, we further compared the immune infiltration score and immune-related function between the high- and low-risk groups in both the TCGA and ICGC cohorts by using ssGSEA. The results showed that the high-risk group was associated with higher levels of immune cell infiltration, including aDCs, DCs, iDCs, pDCs, Macrophages, CD8+ T cells, Th1 cells, Th2 cells, TIL, Treg and Tfh in the TCGA cohort (Figure 8A) as well Treg, aDCs, DCs, iDCs, pDCs, Macrophages, Neutrophils, CD8+ T cells, T helper cells, TIL, Treg, Th1 cells and Th2 cells in the ICGC cohort (Figure 8B). In addition, data from the TCGA cohort suggested that the high-risk group had higher scores in terms of immune checkpoint, inflammation-promoting, APC co-inhibition, APC co-stimulation, T cell co-stimulation, T cell co-inhibition, HLA and MHC class I. Similar results were obtained for data from ICGC cohort. The correlation analysis suggested that risk score was also significantly positively correlated with immune score and stromal score in ICGC cohort $(p<0.001)$, while correlated with immune score in TCGA cohort $(p=0.0011)$.

We further analyzed the correlation between the risk score and the expression level of immune checkpoints in TCGA cohort. The results suggested that risk score was significantly positively correlated with the expression levels of B7-H3, B7H4, TIM-3, LAG3, CTLA-4, BTLA, PD-L1, PD- L2 and PD-1 (Figure 9), 
which combined with TIDE scores implied that patients in high-risk group might be more sensitive to immune checkpoint blockade therapies (Supplementary Figure 4, $p<0.0001$ ).

\section{Discussion}

This study is the first to construct a novel signature based on PRGs in HCC, which can be used to predict prognosis and reflect the immune status for HCC patients. The risk score of PRGs prognostic model combined with traditional TNM staging was proven to be better for predicting the prognosis of HCC in both the discovery TCGA cohort and the validation ICGC cohort.

Pyrolysis that occurs in tumor cells and tumor microenvironment (TME) causes different clinical outcomes through the interactions between pyroptotic cells and $\mathrm{TME}^{[28]}$. On the one hand, as one inflammatory form of programmed cell death, in addition to directly eliminating tumor cells, pyroptosis in tumor cells can also stimulate inflammatory response and reactivate anti-tumor immunity. Wang et al. found that active GSDMA3 could result in immune dependent tumor clearance. HMGB1 released by the pyroptosis of melanoma could activate dendritic cells to enhance the infiltration of CD8+ and CD4+ T cells to inhibit tumor progression and prolong survival ${ }^{[29]}$. The induction of pyroptosis could improve the efficacy of immune checkpoint therapy ${ }^{[30]}$. On the other hand, some of the key components in pyroptosis, such as NLRP3 inflammasome and Gasdermin family proteins, can promote the proliferation, migration and the epithelial-stromal transformation of tumor cells and correlate with poor prognosis ${ }^{[31][32]}$. Various studies proved that IL-18/IL-1 $\beta$ released by pyroptotic tumor cells can induce the proliferation, immunosuppression, angiogenesis and metastasis of varieties of tumors ${ }^{[33][34]}$. The proinflammatory factor HMGB1 produced by GSDME-mediated pyroptosis process promotes the proliferation of colon cancer via activating the ERK $1 / 2$ pathway ${ }^{[35]}$. Pyroptosis-released factors from tumor cells also can again trigger pyroptosis of macrophages, leading to cytokine release and subsequent cytokine release syndrome ${ }^{[36]}$. Further, adjacent normal cells stimulated by inflammatory factors released by pyroptosis may transform into tumor cells ${ }^{[37]}$. In recent years, some researches focus on the notion that chronic inflammation and tumor necrosis related to pyroptosis favor tumor progression. However, the PRGs signature as prognostic model for HCC has not been reported.

In this study, we first compared the expression of 90 PRGs in HCC tissues and adjacent peritumoral tissue in the TCGA cohort. Then, 65 differentially expressed PRGs were screened out and analyzed for their relationship with overall survival. We also found that HCC patients could be clustered into two groups based on the 65 differentially expressed PRGs. In addition, there were significant differences in the clinicopathological characteristics between different pyroptosis clusters, such as tumor stage, tumor size, and survival, which suggesting that the heterogeneity of pyroptosis in tumors may results in different clinical outcomes. Next, we constructed a prognostic model that integrates 8 PRGs through LASSO regression analysis and found that the OS of patients in different risk subgroups is significantly different, both in the discovery set and external validation set. Furthermore, Multivariate Cox regression analysis showed that risk score was an independent predictor for OS. 
The eight PRGs that constitute the prognostic model in our study include HSP90AA1, CHMP4B, BAK1, HSP90AB1, GSDMC, GSDME, STK4 and NOD2, all of which were expressed significantly higher in HCC tissues compared to normal tissues and associated with poor prognosis. Both HSP90AA1 and HSP90AB1 are members of HSP90 family, which has been considered promoting the formation and progression of cancer via varieties of carcinogenic signal pathways ${ }^{[38]}$. In HCC, HSP90 promotes glycolysis and proliferation, and inhibits apoptosis by mediating PKM2 phosphorylation ${ }^{[39]}$. Under hypoxia, the transcription of GSDMC was enhanced to switch TNFa-induced apoptosis to pyroptosis in cancer cells and correlated with poor survival ${ }^{[40]}$. It's been demonstrated that miltirone inhibited HCC cells growth through GSDME-dependent pyroptosis ${ }^{[41]}$, which seemed to contradict the results from TCGA data base. It may be explained by the reason that different levels of GSDME play different roles in HCC development, but the underlying mechanism remains to be addressed. BAK is a key regulator of mitochondria-mediated apoptosis and identified as an oncogenic regulator of HCC. Previous studies suggested BAK plays a promoting role in the stage of hepatocarcinogenesis. BAK deficiency inhibited hepatocyte apoptosis and reduce the tumorigenic rate of liver cancer ${ }^{[42]}$. CHMP4B, a subunit of the endosomal sorting complex required for transport-III, plays an important role in the budding and scission of membrane vesicles of the cell and the late stage of mitotic cell division ${ }^{[43]}$. NOD2, one of the nummber of intracellular PRRs, exaggerates the inflammatory response through RIP2 signaling and promotes hepatocarcinogenesis in mice. The overexpression of NOD2 in HCC also impairs DNA repair through lamin A/C degradation and correlated with poor prognosis of HCC patients ${ }^{[44]}$. STK4, as known as MST1, is a serine/threonine kinase in the Hippo signaling pathway, which role in cancer is yet well illuminated. Recent studies show that functions of Hippo signaling changes with temporal and spatial contexts ${ }^{[45]}$. In our study, both the TCGA and ICGA database suggested that STK4 expression is higher in tumor tissues than that in normal tissues, and positively correlated with poor prognosis. It may be explained by the spatiotemporal heterogeneity of STK4, which has a dynamic expression pattern and play different roles at different stages of cancer.

We then preformed GSEA analysis to explore the potential biological processes and pathways associated with these PRGs. Some representing oncogenic pathways were significantly enriched in high-risk group, including MAPK, VEGF, WNT, TGF- $\beta$, NOTCH signalings. In addition, as expected, some immune responserelated biological functions and pathways were significantly enriched in high-risk group, suggesting pyroptosis process is accompanied with the secretion of inflammatory factors and the activation of inflammasomes.

The difference between high- and low-risk groups in immune infiltration and immune-related functions further validated that inflammation response in the tumor microenvironment might be affected by the prognostic risk model to make it predictive. As observed from the results, the infiltration levels of DCs and macrophages in tumor tissues in the high-risk group is higher than that in low-risk group. Previous studies have demonstrated that tumor-infiltrating DCs play an immunosuppressive role, and plasmacytoid dendritic cells pDCs produced low amounts of INF- $a$, which facilitates the expansion of regulatory $T$ cells and immune tolerance ${ }^{[46]}$. There also evidence demonstrated that the intratumoral infiltration of 
macrophages promoted HCC growth, metastasis, and resistance to sorafenib ${ }^{[47]}$. The difference of Treg levels between low- and high-risk groups also indicates immune function suppression in the high-risk group, which may response to regulating the overactive inflammatory reactions caused by pyroptosis. Of note, the high-risk group has lower activity of type II IFN response with function of tumor immune surveillance and antitumor mechanisms than that of low-risk group ${ }^{[48]}$, While Type I IFNs principally produced by innate immune cells have no difference between the two groups ${ }^{[49]}$.

Furthermore, in our study, the expression of immune checkpoints, including PD1, PDL1, PDL2, CTLA4, B7H3, B7-H4, TIM-3, LAG3 and BTLA in high-risk group were significantly higher than those of low-risk group, and positively correlated with the risk score ${ }^{[50]}$. This may also be one of the reasons for the impairment of immune functions in the high-risk group and can be used to predict the sensitivity to immunotherapy for HCC patients. Based on these findings, it is reasonable to assume that the poor survival outcome of high-risk HCC group may be caused by attenuated anti-tumor immunity.

\section{Conclusion}

In conclusion, we evaluated differential expression of PRGs between normal and HCC tissues and defined a novel 8-gene prognostic risk model based on the pyroptosis for HCC. Then, we verified their relationship with prognosis and clinical features both in TCGA and ICGC cohorts. Moreover, the model risk score was found to be associated with immune response and infiltration of immune cells in the tumor microenvironment. The specific underlying mechanism of PRGs affecting prognosis and the interaction between pyroptosis and tumor microenvironment need further exploration. Our research established a new gene model for predicting the prognosis of HCC patients and revealed potential effect of PRGs on tumorigenesis and development of HCC, which also provided a valuable insight into the relationship between pyroptosis and immunotherapy in HCC.

\section{Declarations}

\section{Ethics approval and consent to participate}

Not applicable.

\section{Consent for publication}

All the authors consented to the publication of this research.

\section{Availability of data and materials}

The datasets analyzed was acquired from The Cancer Genome Atlas (TCGA) database (https://portal.gdc.cancer.gov/) and International Cancer Genome Consortium (ICGC) database (https://dcc.icgc.org/projects/LIRI-JP). 


\section{Competing interests}

The authors declare that they have no confict of interest.

\section{Authors' contributions}

Yi-qun Jin and Xue-song Bai contributed to data analysis, drafting and revising the article, All the authors have agreed on the journal to which the article will be submitted and gave final approval of the version to be published.

\section{Acknowledgements}

Thanks to TCGA and ICGC network for collecting and sharing the huge amount of data.

\section{Compliance with Ethical Standards}

\section{Disclosure of potential conflicts of interest}

No conflict of interests.

\section{Research involving Human Participants and/or Animals}

Not applicable.

\section{Informed consent}

Not applicable.

\section{References}

1. Bray F, Ferlay J, Soerjomataram I, Siegel R L, Torre L A, Jemal A (2018) Global cancer statistics 2018: GLOBOCAN estimates of incidence and mortality worldwide for 36 cancers in 185 countries. CA: a cancer journal for clinicians 68:394-424. https://doi.org/10.3322/caac.21492

2. Islami F, Ward E M, Sung H, Cronin K A, Tangka F, Sherman R L, Zhao J, Anderson R N, Henley S J, Yabroff K R, Jemal A, Benard V B (2021) Annual Report to the Nation on the Status of Cancer, Part 1: National Cancer Statistics. Journal of the National Cancer Institute djab131. Advance online publication. https://doi.org/10.1093/jnci/djab131

3. Zhuang W, Sun H, Zhang S, Zhou Y, Weng W, Wu B, Ye T, Huang W, Lin Z, Shi L, Shi K (2021) An immunogenomic signature for molecular classification in hepatocellular carcinoma. Molecular therapy. Nucleic acids 25:105-115. https://doi.org/10.1016/j.omtn.2021.06.024

4. Tan Y, Chen Q, Li X, Zeng Z, Xiong W, Li G, Li X, Yang J, Xiang B, Yi M (2021) Pyroptosis: a new paradigm of cell death for fighting against cancer. Journal of experimental \& clinical cancer research: CR 40:153. https://doi.org/10.1186/s13046-021-01959-x 
5. Lu X, Guo T, Zhang X (2021) Pyroptosis in Cancer: Friend or Foe?. Cancers 13:3620. https://doi.org/10.3390/cancers 13143620

6. Hsu S K, Li C Y, Lin I L, Syue W J, Chen Y F, Cheng K C, Teng Y N, Lin Y H, Yen C H, Chiu C C (2021) Inflammation-related pyroptosis, a novel programmed cell death pathway, and its crosstalk with immune therapy in cancer treatment. Theranostics 11:8813-8835. https://doi.org/10.7150/thno.62521

7. Hu B, Zhang Q, Gao X, Xu K, Tang B (2021) Monitoring the Activation of Caspases-1/3/4 for Describing the Pyroptosis Pathways of Cancer Cells. Analytical chemistry 93:12022-12031. https://doi.org/10.1021/acs.analchem.1c02158

8. Shi J, Zhao Y, Wang K, Shi X, Wang Y, Huang H, Zhuang Y, Cai T, Wang F, Shao F (2015) Cleavage of GSDMD by inflammatory caspases determines pyroptotic cell death. Nature 526:660-665. https://doi.org/10.1038/nature15514

9. Zhou Z, He H, Wang K, Shi X, Wang Y, Su Y, Wang Y, Li D, Liu W, Zhang Y, Shen L, Han W, Shen L, Ding J, Shao F (2020) Granzyme A from cytotoxic lymphocytes cleaves GSDMB to trigger pyroptosis in target cells. Science (New York, N.Y.) 368:eaaz7548. https://doi.org/10.1126/science.aaz7548

10. Sarhan J, Liu B C, Muendlein H I, Li P, Nilson R, Tang A Y, Rongvaux A, Bunnell S C, Shao F, Green D R, Poltorak A (2018) Caspase-8 induces cleavage of gasdermin D to elicit pyroptosis during Yersinia infection. Proceedings of the National Academy of Sciences of the United States of America 115:E10888-E10897. https://doi.org/10.1073/pnas.1809548115

11. Xia X, Wang X, Cheng Z, Qin W, Lei L, Jiang J, Hu J (2019) The role of pyroptosis in cancer: procancer or pro-"host"?. Cell death \& disease 10:650. https://doi.org/10.1038/s41419-019-1883-8

12. Jiang M, Qi L, Li L, Wu Y, Song D, Li Y (2021) Caspase-8: A key protein of cross-talk signal way in "PANoptosis" in cancer. International journal of cancer 149:1408-1420. https://doi.org/10.1002/ijc.33698

13. Sharma B R, Kanneganti T D (2021) NLRP3 inflammasome in cancer and metabolic diseases. Nature immunology 22:550-559. https://doi.org/10.1038/s41590-021-00886-5

14. Tsuchiya K (2021) Switching from Apoptosis to Pyroptosis: Gasdermin-Elicited Inflammation and Antitumor Immunity. International journal of molecular sciences 22:426. https://doi.org/10.3390/ijms22010426

15. Wu D, Wang S, Yu G, Chen X (2021) Cell Death Mediated by the Pyroptosis Pathway with the Aid of Nanotechnology: Prospects for Cancer Therapy. Angewandte Chemie (International ed. in English) 60:8018-8034. https://doi.org/10.1002/anie.202010281

16. Bedoui S, Herold M J, Strasser A (2020) Emerging connectivity of programmed cell death pathways and its physiological implications. Nature reviews. Molecular cell biology 21:678-695. https://doi.org/10.1038/s41580-020-0270-8

17. Hamarsheh S, Zeiser R (2020) NLRP3 Inflammasome Activation in Cancer: A Double-Edged Sword. Frontiers in immunology 11:1444. https://doi.org/10.3389/fimmu.2020.01444 
18. Ruan J, Wang S, \& Wang J (2020) Mechanism and regulation of pyroptosis-mediated in cancer cell death. Chemico-biological interactions 323:109052. https://doi.org/10.1016/j.cbi.2020.109052

19. Schwarzer R, Laurien L, Pasparakis M (2020) New insights into the regulation of apoptosis, necroptosis, and pyroptosis by receptor interacting protein kinase 1 and caspase-8. Current opinion in cell biology 63:186-193. https://doi.org/10.1016/j.ceb.2020.02.004

20. Hachim M Y, Khalil B A, Elemam N M, Maghazachi A A (2020) Pyroptosis: The missing puzzle among innate and adaptive immunity crosstalk. Journal of leukocyte biology 108:323-338. https://doi.org/10.1002/JLB.3MIR0120-625R

21. Liang F, Zhang F, Zhang L, Wei W (2020) The advances in pyroptosis initiated by inflammasome in inflammatory and immune diseases. Inflamm Res 69:159-166. https://doi.org/10.1007/s00011-02001315-3

22. Gong W, Shi Y, Ren J (2020) Research progresses of molecular mechanism of pyroptosis and its related diseases. Immunobiology 225:151884. https://doi.org/10.1016/j.imbio.2019.11.019

23. Xue Y, Enosi Tuipulotu D, Tan W H, Kay C, Man S M (2019) Emerging Activators and Regulators of Inflammasomes and Pyroptosis. Trends in immunology 40:1035-1052. https://doi.org/10.1016/j.it.2019.09.005

24. Frank D, Vince J E (2019) Pyroptosis versus necroptosis: similarities, differences, and crosstalk. Cell death and differentiation 26:99-114. https://doi.org/10.1038/s41418-018-0212-6

25. Gao J, Aksoy B A, Dogrusoz U, Dresdner G, Gross B, Sumer S O, Sun Y, Jacobsen A, Sinha R, Larsson E, Cerami E, Sander C, Schultz N (2013) Integrative analysis of complex cancer genomics and clinical profiles using the cBioPortal. Science signaling 6:pl1. https://doi.org/10.1126/scisignal.2004088

26. Subramanian A, Tamayo P, Mootha V K, Mukherjee S, Ebert B L, Gillette M A, Paulovich A, Pomeroy S L, Golub T R, Lander E S, Mesirov J P (2005) Gene set enrichment analysis: a knowledge-based approach for interpreting genome-wide expression profiles. Proceedings of the National Academy of Sciences of the United States of America 102:15545-15550.

https://doi.org/10.1073/pnas.0506580102

27. Kanehisa M, Furumichi M, Sato Y, Ishiguro-Watanabe M, Tanabe M (2021) KEGG: integrating viruses and cellular organisms. Nucleic acids research 49:D545-D551.

https://doi.org/10.1093/nar/gkaa970

28. Hou J, Hsu J M, Hung M C (2021) Molecular mechanisms and functions of pyroptosis in inflammation and antitumor immunity. Molecular cell S1097-2765(21)00737-1. Advance online publication. https://doi.org/10.1016/j.molcel.2021.09.003

29. Erkes D A, Cai W, Sanchez I M, Purwin T J, Rogers C, Field C O, Berger A C, Hartsough E J, Rodeck U, Alnemri E S, Aplin A E (2020). Mutant BRAF and MEK Inhibitors Regulate the Tumor Immune Microenvironment via Pyroptosis. Cancer discovery 10:254-269. https://doi.org/10.1158/21598290.CD-19-0672

30. Jaime-Sánchez P, Catalán E, Uranga-Murillo I, Aguiló N, Santiago L, M Lanuza P, de Miguel D, A Arias M, Pardo J (2018) Antigen-specific primed cytotoxic T cells eliminate tumour cells in vivo and 
prevent tumour development, regardless of the presence of anti-apoptotic mutations conferring drug resistance. Cell death and differentiation 25:1536-1548. https://doi.org/10.1038/s41418-018-01129

31. Gao J, Qiu X, Xi G, Liu H, Zhang F, Lv T, Song Y (2018) Downregulation of GSDMD attenuates tumor proliferation via the intrinsic mitochondrial apoptotic pathway and inhibition of EGFR/Akt signaling and predicts a good prognosis in non-small cell lung cancer. Oncology reports 40:1971-1984. https://doi.org/10.3892/or.2018.6634

32. Wang H, Wang Y, Du Q, Lu P, Fan H, Lu J, Hu R (2016) Inflammasome-independent NLRP3 is required for epithelial-mesenchymal transition in colon cancer cells. Experimental cell research 342:184-192. https://doi.org/10.1016/j.yexcr.2016.03.009

33. Deswaerte V, Nguyen P, West A, Browning A F, Yu L, Ruwanpura S M, Balic J, Livis T, Girard C, Preaudet A, Oshima H, Fung K Y, Tye H, Najdovska M, Ernst M, Oshima M, Gabay C, Putoczki T, Jenkins B J (2018) Inflammasome Adaptor ASC Suppresses Apoptosis of Gastric Cancer Cells by an IL18-Mediated Inflammation-Independent Mechanism. Cancer research 78:1293-1307. https://doi.org/10.1158/0008-5472.CAN-17-1887

34. Fathima Hurmath K, Ramaswamy P, Nandakumar D N (2014) IL-1 $\beta$ microenvironment promotes proliferation, migration, and invasion of human glioma cells. Cell biology international 38:14151422. https://doi.org/10.1002/cbin.10353

35. Tan G, Huang C, Chen J, Zhi F (2020) HMGB1 released from GSDME-mediated pyroptotic epithelial cells participates in the tumorigenesis of colitis-associated colorectal cancer through the ERK $1 / 2$ pathway. Journal of hematology \& oncology 13:149. https://doi.org/10.1186/s13045-020-00985-0

36. Liu Y, Fang Y, Chen X, Wang Z, Liang X, Zhang T, Liu M, Zhou N, Lv J, Tang K, Xie J, Gao Y, Cheng F, Zhou Y, Zhang Z, Hu Y, Zhang X, Gao Q, Zhang Y, Huang B (2020)Gasdermin E-mediated target cell pyroptosis by CAR T cells triggers cytokine release syndrome. Science immunology 5:eaax7969. https://doi.org/10.1126/sciimmunol.aax7969

37. Karki R, Kanneganti T D (2019) Diverging inflammasome signals in tumorigenesis and potential targeting. Nature reviews. Cancer 19:197-214. https://doi.org/10.1038/s41568-019-0123-y

38. Xiao H, Wang B, Xiong H X, Guan J F, Wang J, Tan T, Lin K, Zou S B, Hu Z G, Wang K (2021) A novel prognostic index of hepatocellular carcinoma based on immunogenomic landscape analysis. Journal of cellular physiology 236:2572-2591. https://doi.org/10.1002/jcp.30015

39. Xu Q, Tu J, Dou C, Zhang J, Yang L, Liu X, Lei K, Liu Z, Wang Y, Li L, Bao H, Wang J, Tu K (2017) HSP90 promotes cell glycolysis, proliferation and inhibits apoptosis by regulating PKM2 abundance via Thr-328 phosphorylation in hepatocellular carcinoma. Molecular cancer 16:178. https://doi.org/10.1186/s12943-017-0748-y

40. Hou, J, Zhao R, Xia W, et al (2020) PD-L1-mediated gasdermin C expression switches apoptosis to pyroptosis in cancer cells and facilitates tumour necrosis. Nature cell biology 22:1264-1275. https://doi.org/10.1038/s41556-020-0575-z 
41. Zhang X, Zhang P, An L, Sun N, Peng L, Tang W, Ma D, Chen J (2020) Miltirone induces cell death in hepatocellular carcinoma cell through GSDME-dependent pyroptosis. Acta pharmaceutica Sinica B, 10:1397-1413. https://doi.org/10.1016/j.apsb.2020.06.015

42. Hikita H, Kodama T, Shimizu S, Li W, Shigekawa M, Tanaka S, Hosui A, Miyagi T, Tatsumi T, Kanto T, Hiramatsu N, Morii E, Hayashi N, Takehara T (2012) Bak deficiency inhibits liver carcinogenesis: a causal link between apoptosis and carcinogenesis. Journal of hepatology 57:92-100. https://doi.org/10.1016/j.jhep.2012.01.027

43. Wollert T, Wunder C, Lippincott-Schwartz J, Hurley J H (2009) Membrane scission by the ESCRT-III complex. Nature 458:172-177. https://doi.org/10.1038/nature07836

44. Zhou Y, Hu L, Tang W, Li D, Ma L, Liu H, Zhang S, Zhang X, Dong L, Shen X, Chen S, Xue R, Zhang S (2021) Hepatic NOD2 promotes hepatocarcinogenesis via a RIP2-mediated proinflammatory response and a novel nuclear autophagy-mediated DNA damage mechanism. Journal of hematology \& oncology 14:9. https://doi.org/10.1186/s13045-020-01028-4

45. Mirus J E, Zhang Y, Hollingsworth M A, Solan J L, Lampe P D, Hingorani S R (2014) Spatiotemporal proteomic analyses during pancreas cancer progression identifies serine/threonine stress kinase 4 (STK4) as a novel candidate biomarker for early stage disease. Molecular \& cellular proteomics: MCP 13:3484-3496. https://doi.org/10.1074/mcp.M113.036517

46. Sisirak V, Faget J, Gobert M, Goutagny N, Vey N, Treilleux I, Renaudineau S, Poyet G, Labidi-Galy S I, Goddard-Leon S, Durand I, Le Mercier I, Bajard A, Bachelot T, Puisieux A, Puisieux I, Blay J Y, Ménétrier-Caux C, Caux C, Bendriss-Vermare N (2012) Impaired IFN-a production by plasmacytoid dendritic cells favors regulatory T-cell expansion that may contribute to breast cancer progression. Cancer research 72:5188-5197. https://doi.org/10.1158/0008-5472.CAN-11-3468

47. Zhou S L, Zhou Z J, Hu Z Q, Huang X W, Wang Z, Chen E B, Fan J, Cao Y, Dai Z, Zhou J (2016) TumorAssociated Neutrophils Recruit Macrophages and T-Regulatory Cells to Promote Progression of Hepatocellular Carcinoma and Resistance to Sorafenib. Gastroenterology 150:1646-1658.e17. https://doi.org/10.1053/j.gastro.2016.02.040

48. Castro F, Cardoso A P, Gonçalves R M, Serre K, Oliveira M J (2018) Interferon-Gamma at the Crossroads of Tumor Immune Surveillance or Evasion. Frontiers in immunology 9:847. https://doi.org/10.3389/fimmu.2018.00847

49. Negishi $H$, Taniguchi T, Yanai H (2018) The Interferon (IFN) Class of Cytokines and the IFN Regulatory Factor (IRF) Transcription Factor Family. Cold Spring Harbor perspectives in biology 10:a028423. https://doi.org/10.1101/cshperspect.a028423

50. Xu F, Jin T, Zhu Y, Dai C (2018) Immune checkpoint therapy in liver cancer. Journal of experimental \& clinical cancer research: CR 37:110. https://doi.org/10.1186/s13046-018-0777-4

\section{Figures}



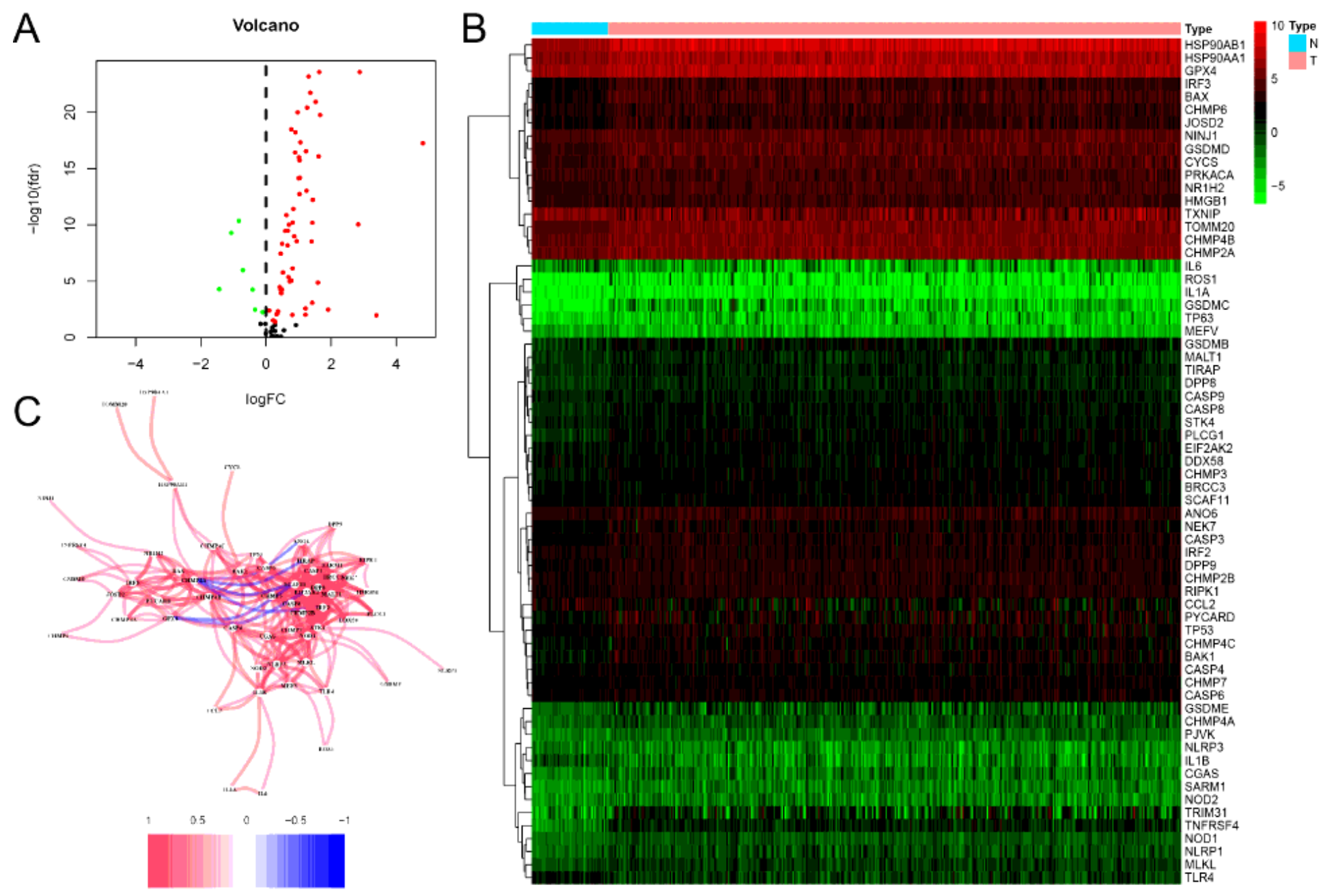

\section{Figure 1}

Expression profile of the candidate 90 PRGs and the interactions among them A. Volcano plot for identification of differentially expressed 65 pyroptosis-related genes from the total of 90 PRGs. Red represents high expression, green represents low expression, black represents no difference between HCC and normal tissues. B. Heatmap (green, low expression level; red, high expression level) of the differentially expressed pyroptosis-related genes between the normal $(N$, blue) and the tumour tissues $(T$, red). C. The correlation network of the differently expressed 65 genes. 


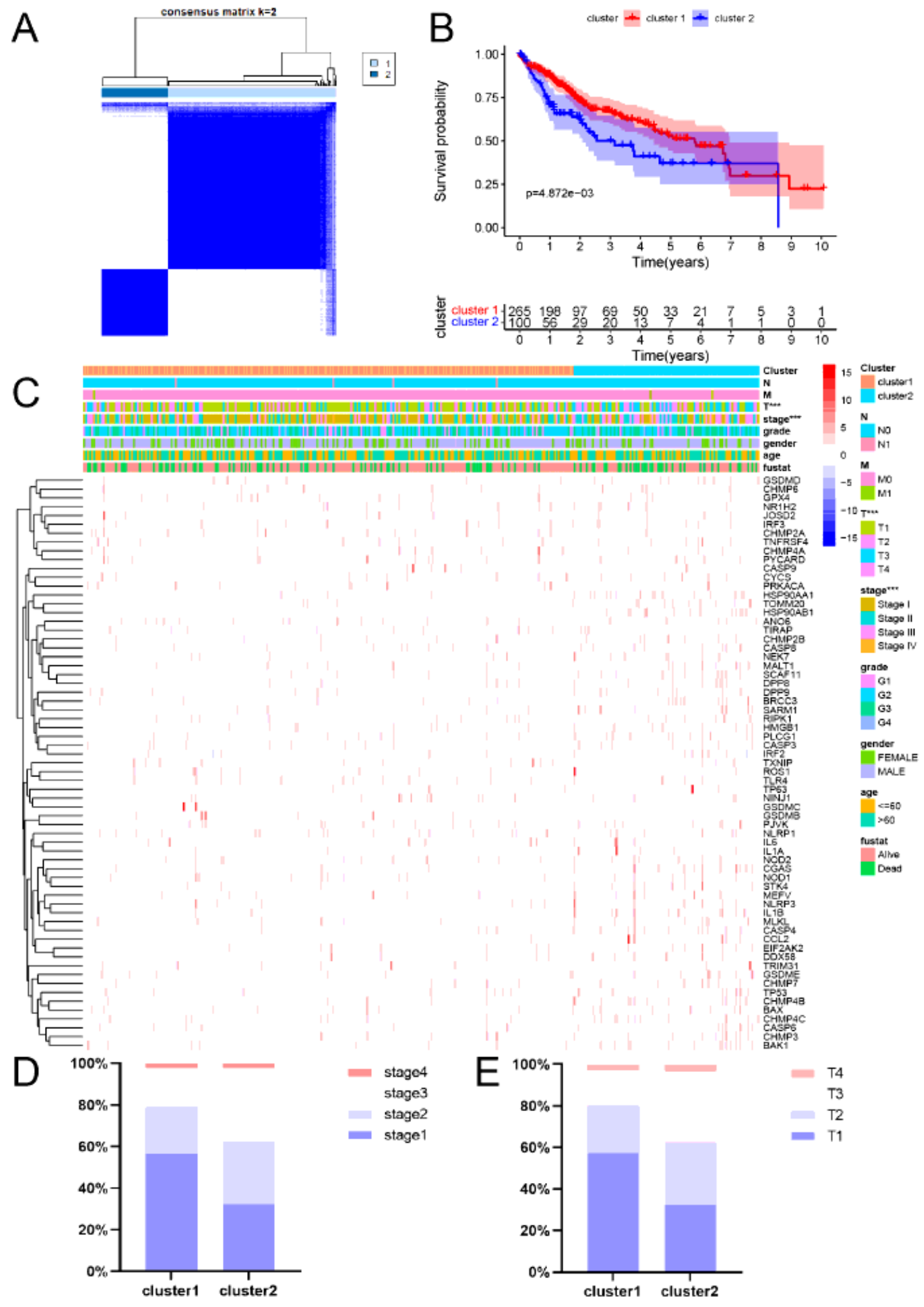

Figure 2

Classification of HCC based on differentially expressed PRGs A. 379 HCC patients were grouped into two clusters by the consensus clustering matrix $(k=2)$. B. KM curves showing the OS difference of cluster 1 (red) and cluster 2 (blue). $p=.0 .004872$. C. Heatmap and the clinicopathologic characters of the two clusters (G1, G2, G3 and G4 are the degree of tumour differentiation. T, N, and M are the Tumor Node 
Metastasis classification) $(* \star \star p<0.001)$. D-E. Stacked graph showing the differences in clinical stage and tumor size between the two clusters $(p<0.001)$.
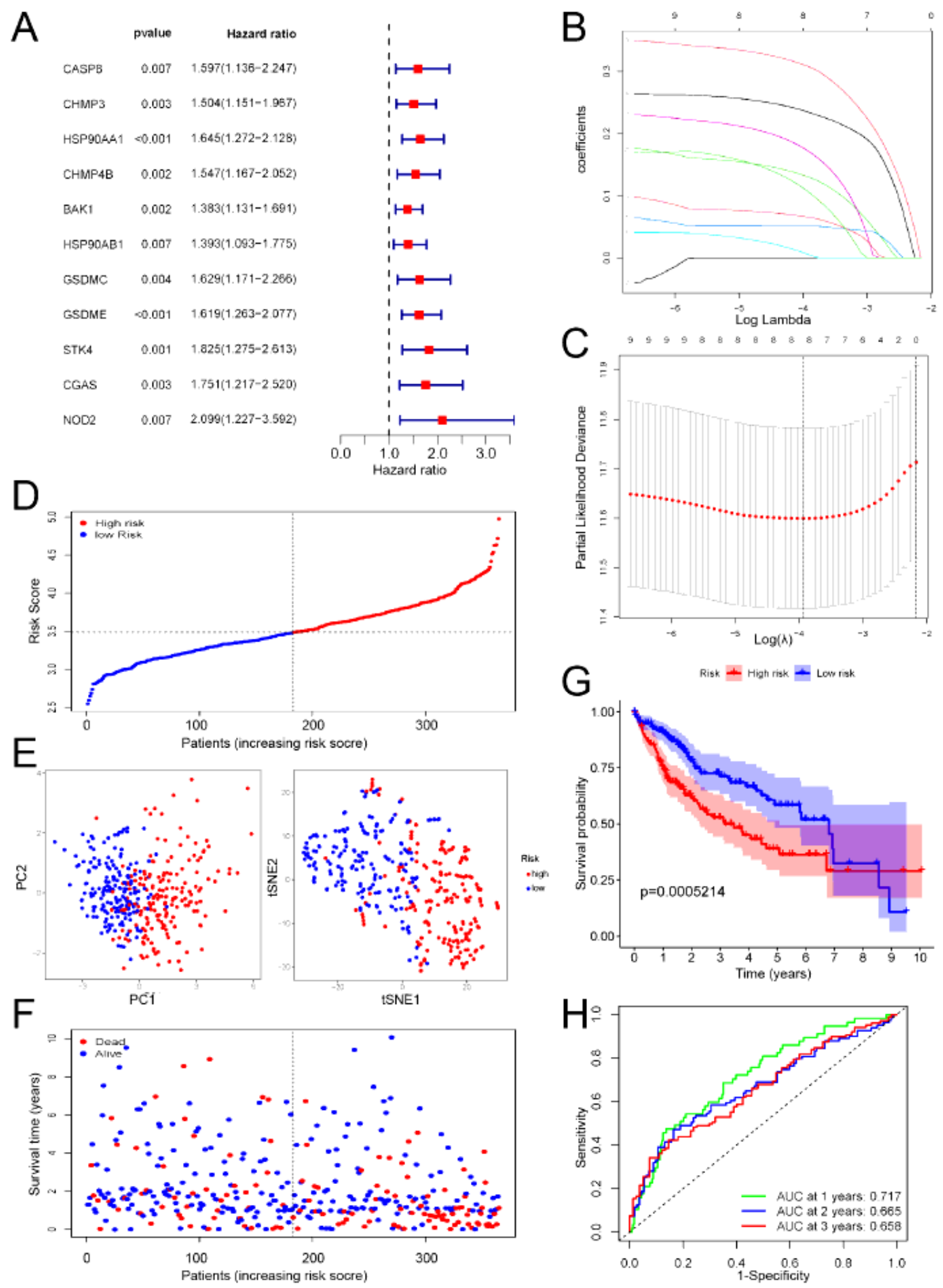

Figure 3

Construction of the PRGs-based prognostic risk model in the TCGA cohort A. Forest plots showing the association between 11 genes expression and OS by univariate cox regression analysis $p<0.01$. B. LASSO-Cox regression analysis for the 11 prognosis related genes. C. Cross-validation for tuning the 
parameter selection in the LASSO regression. D. Distribution of HCC patients based on the median value of risk score. E. PCA plot and t-SNE analysis for validating the distribution of patients based on the risk score. F. The distribution of OS status for each patient. G. Kaplan-Meier curves for comparing the OS of HCC patients between the high- and low-risk groups. The OS of high-risk group is poorer than that of lowrisk group. $p=0.0005214$. H. ROC curves for testing the predictive efficiency of the risk score.
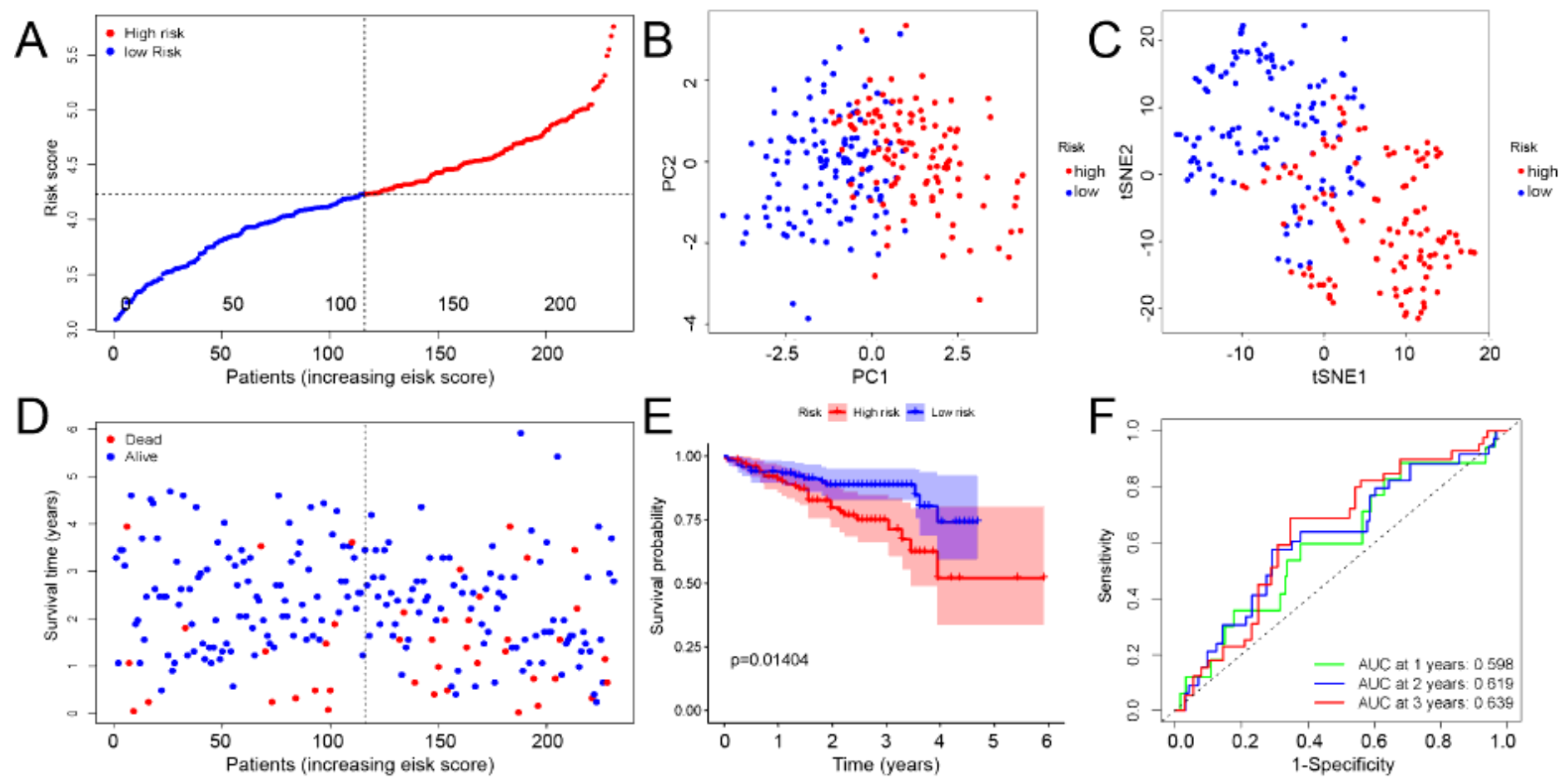

\section{Figure 4}

Validation of the PRGs-based prognostic risk model in the ICGC cohort A. Risk score distribution in HCC patients. B-C. PCA plot and t-SNE analysis. D. Overall survival and survival status of HCC patients in the ICGC database. E. Compared to the high-risk groups, patients in low-risk groups have better OS outcomes. $p=0.01404$. F. The ROC curves of the risk score in HCC. 

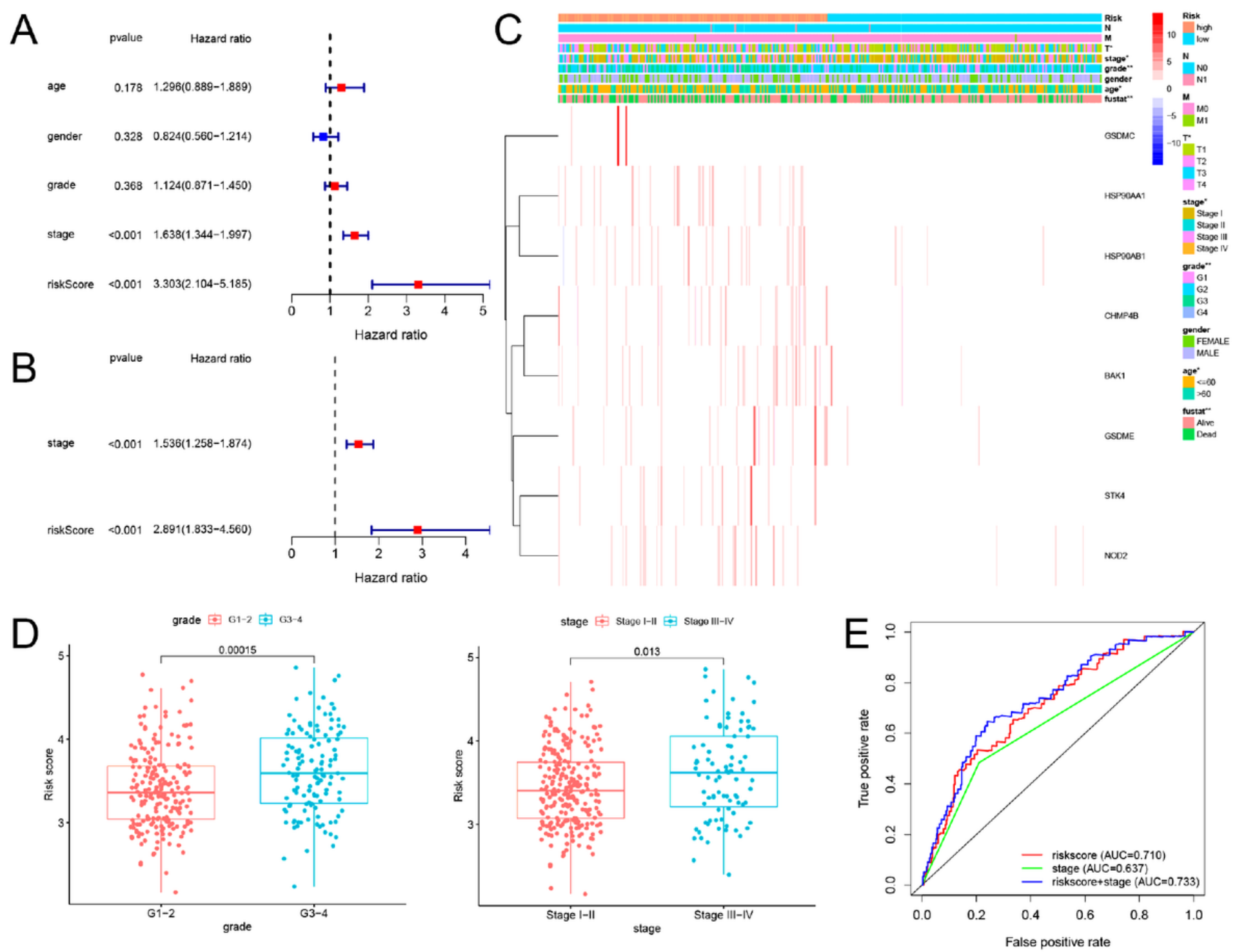

Figure 5

Prognostic Value of the Prognostic Risk Model A. Univariate analysis for OS-related factors. B. Multivariate analysis for OS-related factors. C. Heatmap and the clinical characters of low- and high-risk groups (G1, G2, G3 and G4 are the degree of tumour differentiation. T, N, and M are the Tumor Node Metastasis classification) $\left({ }^{*} p<0.05,{ }^{*} p<0.01\right)$. D. The risk score in different groups divided by clinical characteristics (grade: on the lift panel, stage: on the right panel). E. Time-dependent ROC curve was used to compare the prognostic accuracy of risk score, tumor stage, and the combination of risk score and tumor stage. 

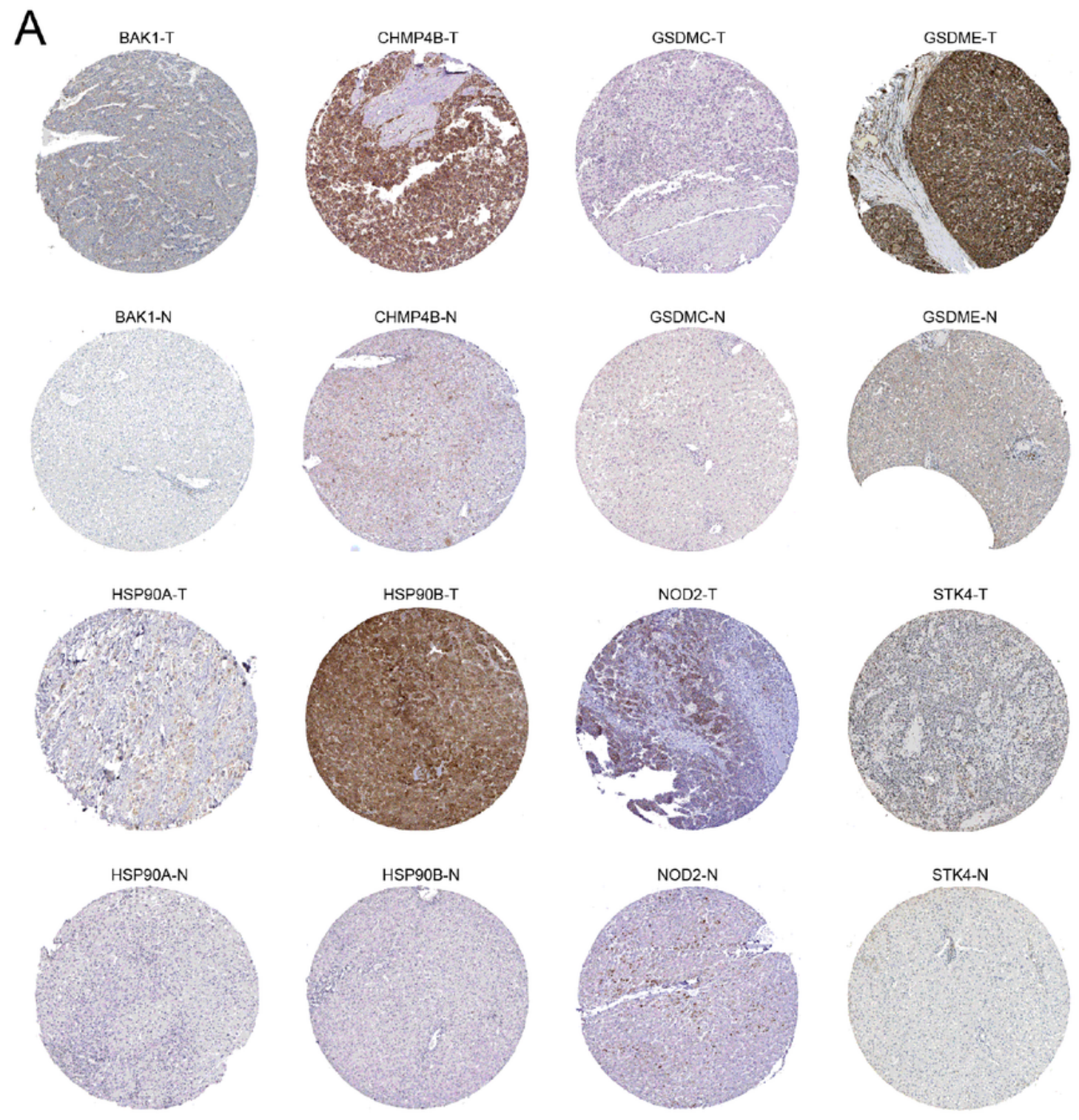

B

HS9oAas $1.9 \%$ | |

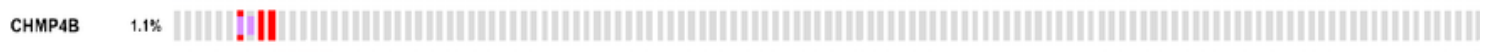

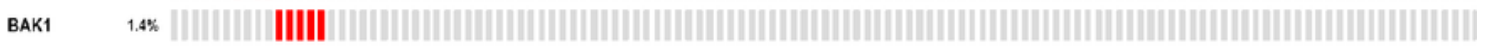
HS99ose s. Gsomc gsome

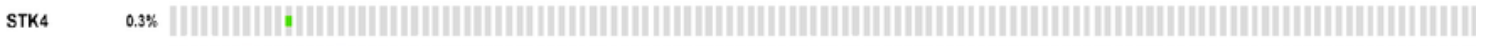

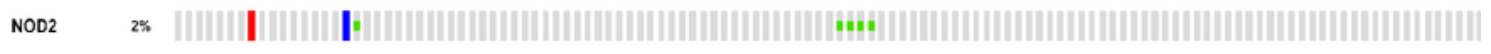
Genete Alemertion

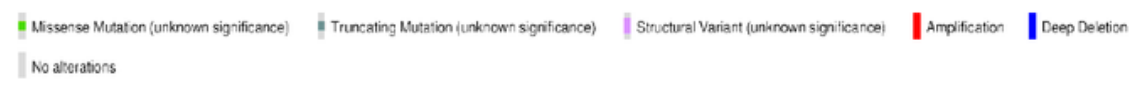

Figure 6

Expression and Mutation profiles of Prognostic Genes Between HCC Tissues and Para-carcinoma Tissues A. Immunohistochemical staining of the expression levels of 8 PRGs involved in the model between HCC and para-carcinoma tissues according to the Human Protein Atlas (HPA) database. B. Visualization of the mutation data for 8 PRGs in $353 \mathrm{HCC}$ samples using the cBioPortal. 

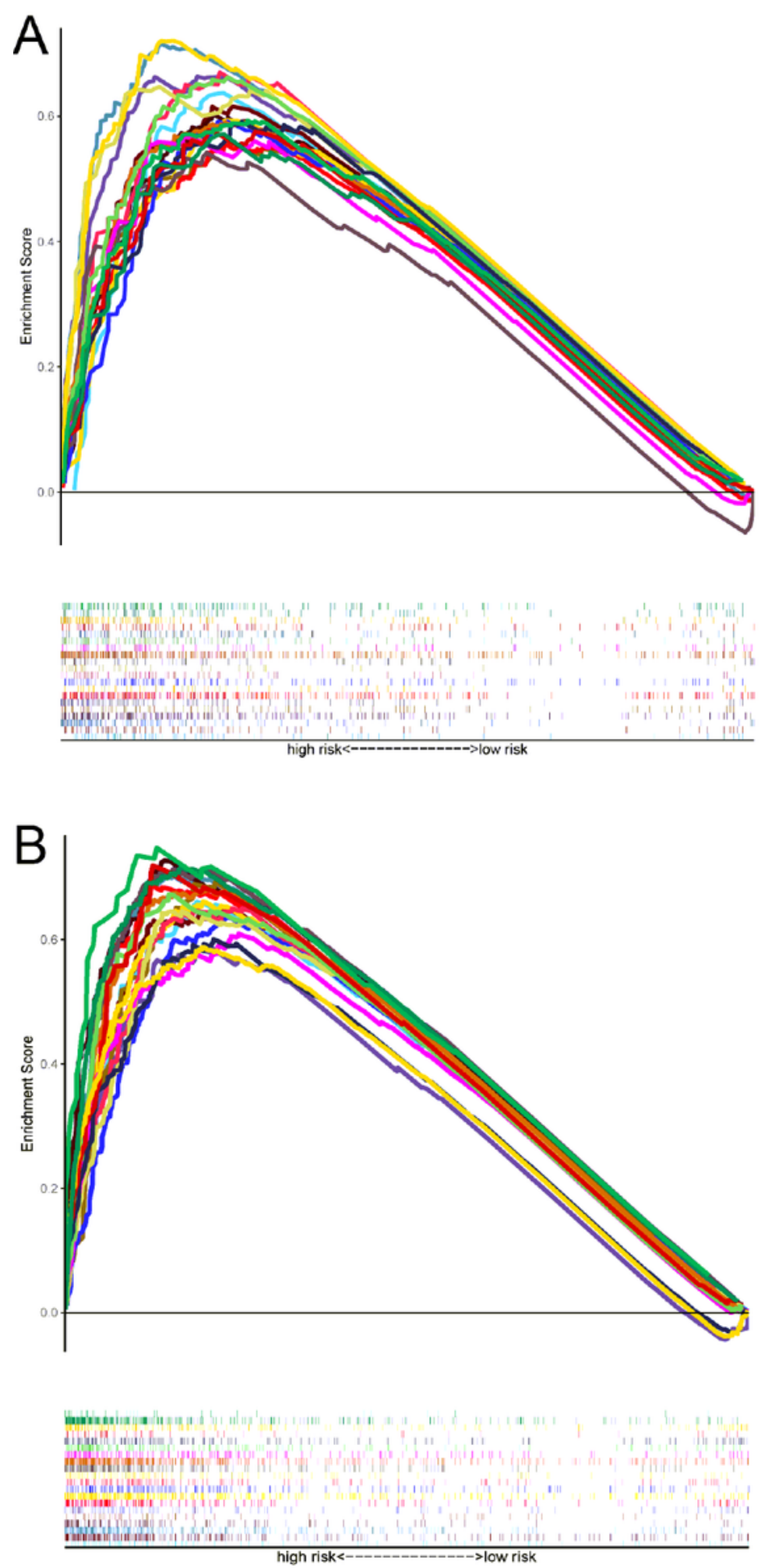

\section{Figure 7}

Gene set enrichment analysis of GO (A) and KEGG (B)
- KEgG B CELL RECEPTOR SIGNaling PATHWAY

- KEGG_CELL_CYCLE

- KEGG CHEMOKINE SIGNAIING PATHWAY

- KEGG FC EPSILON RI SIGNALING PATHWAY

- KEGG FC GAMMA R MEDIATED PHAGOCYTOSIS

- KEGG_MAPK SIGNAL ING PATHWAY

- KEGG_MTOR_SIGNALING_PATHWAY

- KEGG_NATURAL_KILLER_CELL_MEDLATED_CYTOTOXICITY

- KEGG_NOD_LIKE_RECEPTOR_SIGNALING_PATHWAY

- KEGG_NOTCH_SIGNALING_PATHWAY

- KEGG_P53 SIGNALING_PATHWAY

- KEGG pathwars In Cancer

- KEGG RIG I LIKE RECEPTOR SIGNALING PATHWAY

- KEGG_T_CELL_RECEPTOR_SIGNALING_PATHWAY

- KEGG_TGF_BETA_SIGNALING_PATHWAY

- KEGG_TOLL_LIKE_RECEPTOR_SIGNALING_PATHWAY

- KEGG_UBIQUITIN_MEDIATED_PROTEOLYSIS (1)

- KEGG_VEGF_SIGNALING_PAIHWAY

- KEGG WNT SIGNALING PATHWAY
- GO_CELL_AGING

- GO_CELL_CYCLE_G1_S_PHASE_TRANSITION

- GO_CELL_CYCLE_G2_M_PHASE_TRANSITION

- GO_CELLULAR_RESPONSE_TO_HEAT

- go_CELLULAR_SEnESCENCE

- go chaperone BINDING

- GO_IMPORT_INTO_NUCLEUS

- GO_NEGATIVE_REGULATION_OF_MITOTIC_CELL_CYCLE

- GO_NIK_NF_KAPPAB_SIGNALING

- GO_NON_CANONICAL_WNT_SIGNALING_PATHWAY

- GO_POSITIVE_REGULATION_OF_WNT_SIGNALING_PATHWAY

- GO_REGULATION_OF_CELL_CYCLE_G2_M_PHASE_TRANSITION

- GO_REGULATION_OF_CELL_CYCLE_PHASE_TRANSITION

- GO REGULATION_OF_INTRINSIC_APOPTOTIC SIGNAUNG PATHWAY

- GO_REGULATION_OF_MACROAUTOPHAGY

- GO_REGULATION_OF_MITOCHONDRION_ORGANIZATION

- GO_REGULATION_OF_PROTEIN_IMPORT

- Go_REgULATION_OF_PROTEIN_STABILITY

- GO_RNA_SPLICING_VIA_TRANSESTERIFICATION_REACTIONS

- GO_UBIQUITIN_LIKE_PROTEIN_CONJUGATING_ENZYME_ACTIVITY 

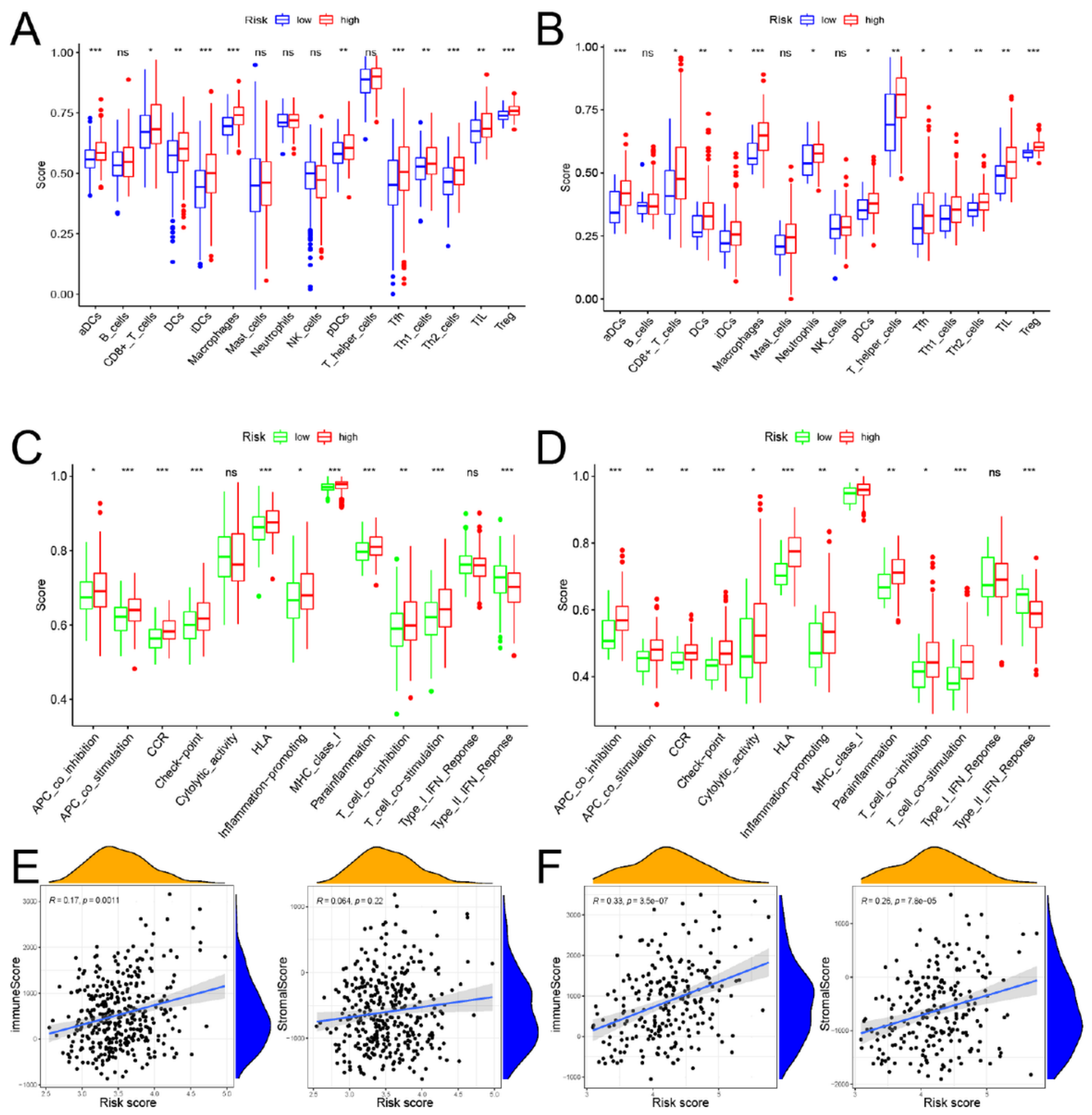

Figure 8

Comparison of the sSGSEA scores of immune cells and immune pathways $A, B$ Comparison of the sSGSEA scores of 16 immune cells between low- (blue) and high-risk (red) group in the TCGA cohort (A) and ICGC cohort (B). C, D Comparison of the sSGSEA scores of 13 immune-related pathways between low- (green) and high-risk (red) group in the TCGA cohort (C) and ICGC cohort (D). E, F. The correlation 
analysis between the risk score and immune score, as well as the risk score and stromal score in TCGA cohort (E) and ICGC cohort (F).
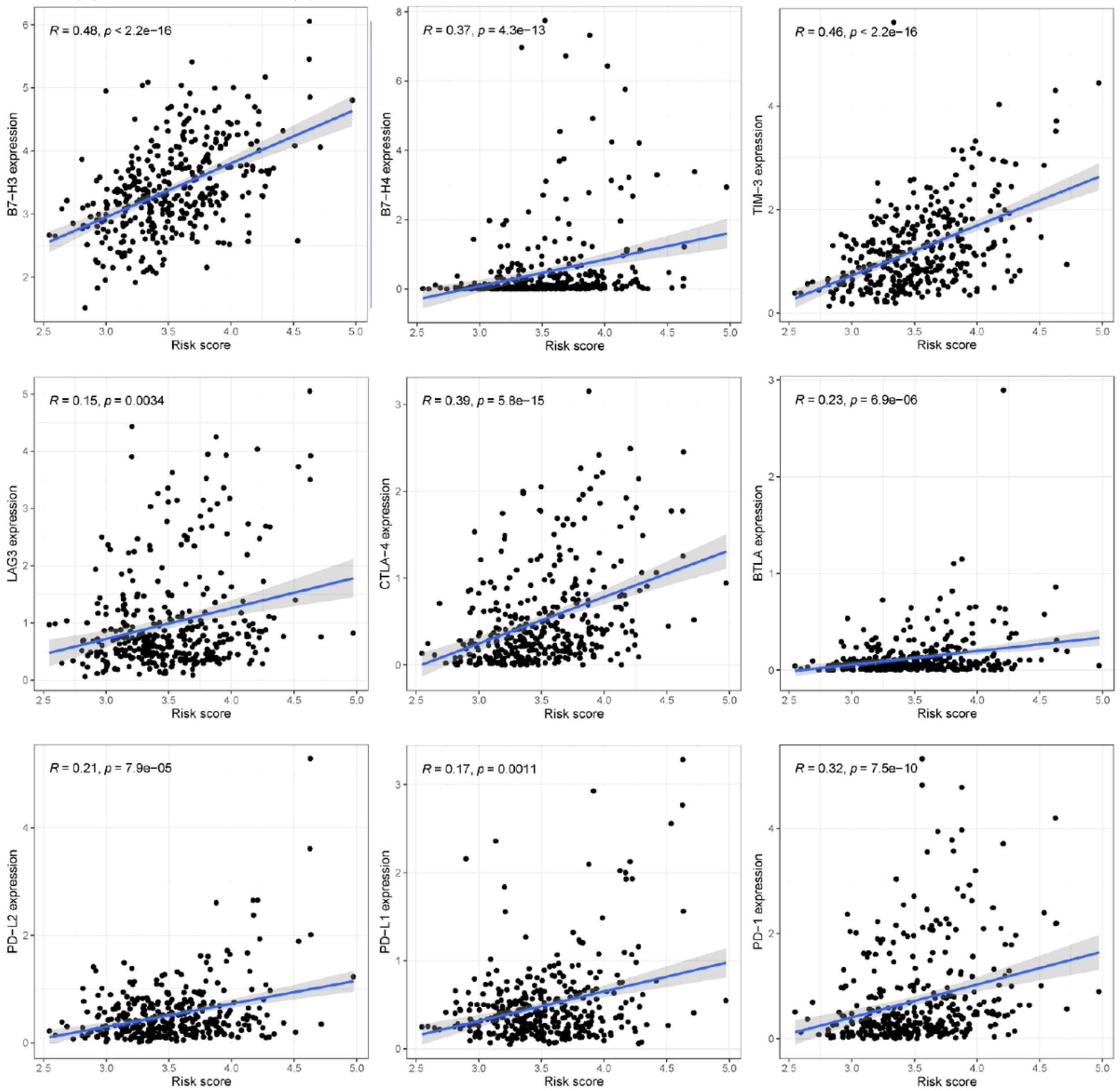

Figure 9

The correlation analysis between risk score and the expression levels of immune checkpoints, such as B7-H3, B7H4, TIM-3, LAG3, CTLA-4, BTLA, PD-L1, PD- L2 and PD-1

\section{Supplementary Files}


This is a list of supplementary files associated with this preprint. Click to download.

- SupplementaryFig1.tif

- SupplementaryFig2.tif

- SupplementaryFig3.tif

- SupplementaryFig4.tif

- SupplementaryTable1.docx 